\title{
A Multicentre Retrospective Case Review of Outcomes and Complications of S53P4 Bioactive Glass in Mastoid Obliteration Surgery.
}

\author{
Colin Leonard ${ }^{1}$, Sinead McNally ${ }^{2}$, Mark Adams ${ }^{1}$, Susie Hampton ${ }^{1}$, Edward McNaboe ${ }^{2}$, C \\ Ekambar Reddy $^{2}$, and Neil Bailie ${ }^{1}$ \\ ${ }^{1}$ Royal Victoria Hospital \\ ${ }^{2}$ Craigavon Area Hospital
}

June 15, 2020

\begin{abstract}
Objective The objective of this multicentre retrospective case review was to assess the natural clinical course, efficacy and safety of Mastoid Obliteration with S53P4 Bioactive Glass (bioactive glass). Study Design Retrospective case note review Setting Regional Tertiary Referral centre and District General Hospital Participants Patients undergoing mastoid cavity obliteration as part of primary or secondary procedure with bioactive Glass between 2012 - 2018. Outcome measures were assessed from a prospectively collated database and case note review. Main Outcome Measures Primary outcomes were the common morbidities of a mastoid cavity; dry or discharging ear (Merchant's scale), vertigo in cold air and a water tight middle ear. Patients were also assessed for audiological outcomes and recidivism. Results 90 patients were included. During the follow up period, (mean $=22$ months; range $=6-59$ months) cholesteatoma recidivism was observed in $2 \%$ of ears, (2 patients). An acceptably dry (Merchant Grade $0-1$ ) ear was achieved in $91 \%$ of all ears (95\% Primary Cases, $80 \%$ Secondary Cases). Delayed healing of the graft in the external ear canal retaining the S53P4BAG Bioactive Glass (BonAlive?,(BonAlive Biomaterials Ltd., Turku, Finland)) within the mastoid occurred in $13 \%$ (12 ears). However, in all cases conservative management resulted in complete healing. Conclusions Bioactive glass provides a safe and effective means of mastoid obliteration. Complications including overlay graft failure and slow epithelisation resulting in prolonged postoperative discharge (up to 2 months) and dehiscence into the external ear canal do not preclude full recovery and may be successfully managed conservatively.
\end{abstract}

Title: A Multicentre Retrospective Case Review of Outcomes and Complications of S53P4 Bioactive Glass in Mastoid Obliteration Surgery.

Running Title: Bioactive glass in mastoid obliteration

\section{Abstract}

Objective

The objective of this multicentre retrospective case review was to assess the natural clinical course, efficacy and safety of Mastoid Obliteration with S53P4 Bioactive Glass (bioactive glass).

Study Design

Retrospective case note review

Setting

Regional Tertiary Referral centre and District General Hospital 


\section{Participants}

Patients undergoing mastoid cavity obliteration as part of primary or secondary procedure with bioactive Glass between 2012 - 2018. Outcome measures were assessed from a prospectively collated database and case note review.

\section{Main Outcome Measures}

Primary outcomes were the common morbidities of a mastoid cavity; dry or discharging ear (Merchant's scale), vertigo in cold air and a water tight middle ear. Patients were also assessed for audiological outcomes and recidivism.

Results

90 patients were included. During the follow up period, (mean $=22$ months; range $=6-59$ months) cholesteatoma recidivism was observed in $2 \%$ of ears, (2 patients). An acceptably dry (Merchant Grade 0 1) ear was achieved in $91 \%$ of all ears (95\% Primary Cases, $80 \%$ Secondary Cases). Delayed healing of the graft in the external ear canal retaining the S53P4BAG Bioactive Glass (BonAlive?,(BonAlive Biomaterials Ltd., Turku, Finland)) within the mastoid occurred in 13\% (12 ears). However, in all cases conservative management resulted in complete healing.

\section{Conclusions}

Bioactive glass provides a safe and effective means of mastoid obliteration. Complications including overlay graft failure and slow epithelisation resulting in prolonged postoperative discharge (up to 2 months) and dehiscence into the external ear canal do not preclude full recovery and may be successfully managed conservatively.

\section{Key Points}

1. A mastoid cavity presents significant morbidity to the patient and burden on the clinical service.

2. Obliteration of the mastoid cavity aims to prevent these problems and improve patients quality of life.

3. Bioactive glass provides a safe and effective means of mastoid obliteration without extensive dissection of a soft tissue flap to fill the defect.

4. The most common complication suffered by patients is an increased frequency of attendance in the initial healing phase as the graft covering the bioactive glass develops a blood supply and epithelises.

5. In our experience bioactive glass can be placed onto dehiscent facial nerve and dura without complication.

\section{Introduction}

The "Front-to-Back" approach to cholesteatoma surgery is widely utilised. It minimises the destruction of disease free mastoid, allows for early surgical identification of the integrity of ossicular chain and other important anatomical structures. Despite these advantages, the resulting canal wall defect presents a challenge to the surgeon. The defect enlarges with extent of surgery from Atticotomy, to Atticoantrostomy and Modified Radical Mastoidectomy; M2a, M2b and M2c in the International Otology Outcomes Group consensus statement.[1] The surgeon must decide upon the management of these defects intraoperatively using their clinical acumen and knowledge of the patient's lifestyle and wishes. There are many different means by which a mastoid cavity may be obliterated. There are a number of techniques that have been utilized to facilitate this outcome: cartilage to reconstruct the canal wall; bone chips harvested intraoperatively, a variety of muscular flaps and synthetic substances to obliterate the mastoid cavity. [2 3$]$

The morbidity to the patient of a mastoid cavity including dizziness on exposure to cold air, being prone to more frequent infections, potential lifelong attendance at outpatient departments, and the associated burden to the service of providing such care, are far from insignificant. Thus the benefits of obliterating the mastoid cavity are to both the patient and the clinical service. In a recent study, obliteration of the mastoid has been shown to reduce disease recurrence in children. [4] 
S53P4BAG Bioactive Glass (BonAlive?,(BonAlive Biomaterials Ltd., Turku, Finland)) (Bioactive Glass), is a silica based biomaterial, composed of Silicon Dioxide, Sodium Oxide, Calcium Oxide, and Phosphorous Pentoxide. Bioactive glass has several properties that are advantageous in mastoid obliteration as it is both osteoconductive (it provides a framework for bone growth), and osteoproductive (it stimulates bone growth).[5] It has also been demonstrated to have antibacterial properties against a wide range of microorganisms. [6-8] There have been several studies published relating to the safety, anatomical, functional and quality of life relating to the use of bioactive glass. [9-11] However, there is a sparsity of evidence relating to the clinical application of bioactive glass, complications and their management. The aim of this retrospective case review was to identify the techniques and outcomes achieved when utilizing bioactive glass to obliterate the mastoid. Data relating to these issues relates to consent, shared decision making with our patients and intraoperative decision making.

\section{Materials and Methods}

\section{Study Design}

Consultant Otolaryngology-Head and Neck Surgeons with a subspecialist interest in Otology working in Northern Ireland were asked to identify patients for this retrospective case review through their prospectivelyrecorded surgical logbook. The search terms "Obliteration," and "Mastoid Obliteration," were, where the respective surgeons questioned the completeness of the data, augmented by a search for all patients who had undergone "Modified Radical Mastoidectomy."

\section{Methods}

Patient notes were identified and reviewed in both physical and electronic health care records. Patients with less than six months clinical follow up were excluded. Patient demographics, surgical details including confirmation of the use of Bioactive glass, presence or absence of complications of open cavity surgery (dry ear (Merchant's scale 0-1, Vertigo in Cold air, Water tight ear), audiological outcomes, and recurrence were recorded in a spreadsheet prior to statistical analysis using R. Studio software (R Core Team, 2018). [12]

Results

Four surgeons, working in two different Otolaryngology departments identified 90 patients who had underwent primary procedures utilising bioactive. The patients had a median age of 38 years (Range 10 years 88 years), with gender and operative side ratio 1:1. Median follow up at time of data collection was 15.5 months (Range 6 months - 65 months).

\section{Surgical Techniques}

Bioactive Glass was used to obliterate mastoid cavities in 55 primary procedures and 35 secondary procedures. 57 ears were approached using a postaural soft tissue approach, 33 via a endaural approach. Table I. shows the classification of cases in both primary and secondary obliteration.

Ossicular status, as recorded in the operative notes, was graded using the Mills Staging System[13], from 0 (Chain intact) to 3 (Erosion malleus, incus and stapes arch). In 23 cases, the ossicular status was not able to be determined. In some cases, this was because the middle ear was not entered during a secondary obliteration $(\mathrm{n}=14)$, or revision surgery, and in others where clear documentation was not available at the time of not review $(n=9)$. Chi-squared testing was performed to look for greater representation of one ossicular status than the others, and none was found $(\mathrm{p}=0.78)$. Table II. shows the number of cases at each ossicular Mills status. 41 patients underwent ossiculoplasty, the most common means of reconstruction being cartilage reinforced Type III $(\mathrm{n}=17)$, or use of Titanium Partial $(\mathrm{n}=12)$ or Total Ossicular Reconstruction Prosthesis $(\mathrm{n}=5)$.

The 7th cranial nerve (Facial) was exposed as a result of disease in 8 cases. A fistula in the lateral semicircular canal was present in 5 cases. A further 7 patients were found to have both exposed Facial nerve and a fistula in the lateral semicircular canal. The presence of both facial dehiscence and lateral semicircular canal fistula in such a high proportion of patients highlights the need for intraoperative vigilance, in particular where 
one dehiscence has already been identified. At the time of publication, no patient had suffered delayed complications as a result of the use of Bioactive glass in the presence of a labyrinthine or facial nerve dehiscence.

A number of different graft materials were used for both the tympanic membrane repair and as a covering for Bioactive Glass. The covering graft prevents the bioactive glass from extruding into the external auditory canal and as an autograft provides a framework for epithelial growth (Figure 1).

The diversity of procedures, ossicular status, and the objective of this paper being to assess the use and clinical outcomes of Bioactive Glass mean that assessment of audiological outcomes by subgroup has not been performed. However, the median change of Air Bone Gap between Pre and Post ( $>6$ months) operative audiometry was $1 \mathrm{~dB}$, (Range $-36 \mathrm{~dB}-+25 \mathrm{~dB}$ ). 31 had change within the margin of error for audiometry $(+/-5 \mathrm{~dB})$, whilst 19 had a lower hearing threshold post operatively and 21 a higher hearing threshold postoperatively.

Clinical complications of open cavity surgery (dependence on clinical staff for aural toilet, discharging cavity, vertigo in cold air, and need to protect the ear from water) are shown in Figure 2. At the time of publication no patient has demonstrated recurrence. However, 6 patients have demonstrated retraction of the attic, one of whom has underwent exploratory tympanotomy where a suspicious area was found to be scar tissue rather than cholesteatoma pearl. Seven patients had intermittently discharging ears at the time of data collection (one as a result of ventilation tube). However, 18 (20\%) (10 Primary, 8 Secondary) reported the presence of discharge and attended the outpatient department frequently for up to 6 weeks post-operatively. This was due to delayed healing of the graft covering of the Bioactive Glass and, in all cases, responded to regular microsuction and the use of topical antibiotic and steroid creams. All healed without deleterious effect on the mastoid obliteration. Further complication was seen in the occurrence of surgical site infection in two patients, both of whom resolved with oral and topical antibiotic treatment. One patient failed to comply with follow up and attended a solitary review at greater than six months post operatively. She had suffered prolonged discharge from the ear that had settled a few weeks prior to review. There had been complete loss of the bioactive glass and covering graft tissue but she had been left with a well healed and dry mastoid cavity.

\section{Discussion}

Within the limits of the current study, Bioactive glass provides a safe and effective means of mastoid obliteration. We report outcomes in a group of patients who have undergone mastoid obliteration as an adjunct to a diverse group of procedures. Complications of the procedure are rare and outcomes good, irrelevant of surgical approach to initial mastoid surgery and concurrent procedures. Whilst concurrent or previous procedures may impact on the choice of covering graft, the most common complication suffered by patients is an increased frequency of attendance in the initial healing phase for ear dressing. This is to facilitate epithelialisation of the graft covering the bioactive glass.

We did not aim to assess the impact of obliteration on hearing outcomes within this retrospective study. Where the ossicular status was recorded, it did not preclude a decision to make use of Bioactive glass, nor did the Bioactive glass preclude the performance of an ossiculoplasty.

Where dehiscence of the lateral semicircular canal had occurred, surgical decision making was uniform in that all patients had a periosteal or fascial graft placed over the dehiscence prior to application of bioactive glass. In contrast in some cases bioactive glass was placed directly upon the dura and facial nerve in the event of their exposure. The duration of follow up is widely varied within our population. However, it is reassuring to note that none of these patients have had any late complications arising as a result of their obliteration during the follow-up period.

The issue of recurrence and recidivism has impacted upon the surgical technique used in obliterating the mastoid cavity. In the first few patients receiving Bioactive glass, the grafts were placed with temporalis fascia or muscular flaps providing both tympanic and covering grafts. In some of these early patients, the attic 
has retracted (Image 1). Our practice takes a number of measures to facilitate a successful obliteration and to attempt to prevent the position of clinical equipoise presented by a new retraction pocket in a previously operated ear. Firstly, when performing the mastoidectomy, the facial ridge is only lowered as far as needs be to ensure disease clearance. This is another advantage to the front-back approach, which was already preferred by surgeons reporting in this paper (Table I.). Secondly, when performing the reconstruction and obliteration, a small attic cavity is lined with a temporalis fascia graft, prior to using tragal cartilage to seal off the mastoid cavity from the attic and middle ear space. The mastoid may then be filled with Bioactive glass and covered appropriately. Once healed, the small attic cavity (Image 2) is easily examined and cleaned, should cleaning prove necessary. The retention of a small attic cavity and high facial ridge reduces the surface area of the covering graft. However, it has not obviated the problem of getting such a large graft to heal in the external ear canal. There have been a number of pedicled flaps described and utilised in mastoid obliteration.[3] The small number of local flaps performed as part of our series precludes comment on the efficacy when utilising Bioactive glass.

The current practices and techniques of surgeons beginning to make use of Bioactive glass, will likely determine both their patient and technique selection. Where a surgeon is confident in their disease clearance, primary obliteration may be undertaken but the long term benefits of reduced attendance at the outpatient department may just as well be achieved through a secondary procedure, at which time disease clearance may be confirmed. In secondary procedures, the potential to utilise the epithelium of the mastoid cavity, with its subdermal blood supply to cover the obliteration, is appealing but often impractical. The choice of grafts and flaps used during the obliteration will also likely depend upon the experience of the individual surgeon and indeed on what is available following previous procedures.

When consenting patients, they may expect that $90 \%$ of patients receive a benefit from the troublesome symptoms of mastoid cavity. However, it is important to inform them of a 1 in 5 chance of prolonged discharge and the need for repeat attendance until healing is complete. Bioactive glass provides a safe and effective means of mastoid obliteration. The role of obliteration in prevention of recurrence, the mechanism of its benefit, and the clinical outcomes achieved require further prospective study.

Figure 1. Frequency of covering graft material in Primary and Secondary Obliteration.

Figure 2. Frequency of common complications of mastoid cavity in patients who have underwent Mastoid Obliteration with Bioactive Glass. (AA - Atticoantrostomy, MRM - Modified Radical Mastoidectomy)

Image 1. Image showing retraction of attic in an early case where the attic was reconstructed with fascia as opposed to cartilage

Image 2. Image captured at endoscopic examination of the ear showing post-operative appearance

Table 1 : Surgery performed in primary and secondary procedures prior to obliteration.

Table 2: Ossicular status of ears undergoing exploration of the middle ear

References

1. Yung M, James A, Merkus P, et al. International Otology Outcome Group and the International Consensus on the Categorization of Tympanomastoid Surgery. J Int Adv Otol 2018;14 (2):216-26 doi: 10.5152/iao.2018.5553[published Online First: Epub Date]|.

2. Kurien G, Greeff K, Gomaa N, Ho A. Mastoidectomy and mastoid obliteration with autologous bone graft: a quality of life study. J Otolaryngol Head Neck Surg 2013;42 :49 doi: 10.1186/1916-0216-42-49[published Online First: Epub Date]|.

3. Yung M, Bennett A. Use of mastoid obliteration techniques in cholesteatoma. Curr Opin Otolaryngol Head Neck Surg 2013;21 (5):455-60 doi: 10.1097/MOO.0b013e3283646521[published Online First: Epub Date]|. 
4. Trinidade A, Skingsley A, Yung MW. Pediatric cholesteatoma surgery using a single-staged canal wall down approach: results of a 5-year longitudinal study. Otol Neurotol 2015;36 (1):82-5 doi: 10.1097/MAO.000000000000598[published Online First: Epub Date]|.

5. Valimaki VV, Aro HT. Molecular basis for action of bioactive glasses as bone graft substitute. Scand J Surg 2006;95 (2):95-102 doi: 10.1177/145749690609500204[published Online First: Epub Date]|.

6. Drago L, De Vecchi E, Bortolin M, Toscano M, Mattina R, Romano CL. Antimicrobial activity and resistance selection of different bioglass $\mathrm{S} 53 \mathrm{P} 4$ formulations against multidrug resistant strains. Future Microbiol 2015;10 (8):1293-9 doi: 10.2217/FMB.15.57[published Online First: Epub Date]|.

7. Lepparanta O, Vaahtio M, Peltola T, et al. Antibacterial effect of bioactive glasses on clinically important anaerobic bacteria in vitro. J Mater Sci Mater Med 2008;19 (2):547-51 doi: 10.1007/s10856-007-3018$5[$ published Online First: Epub Date]|.

8. Munukka E, Lepparanta O, Korkeamaki M, et al. Bactericidal effects of bioactive glasses on clinically important aerobic bacteria. J Mater Sci Mater Med 2008;19 (1):27-32 doi: 10.1007/s10856-007-31431[published Online First: Epub Date]|.

9. Bernardeschi D, Nguyen Y, Russo FY, Mosnier I, Ferrary E, Sterkers O. Cutaneous and Labyrinthine Tolerance of Bioactive Glass S53P4 in Mastoid and Epitympanic Obliteration Surgery: Prospective Clinical Study. Biomed Res Int 2015;2015 242319 doi: 10.1155/2015/242319[published Online First: Epub Date]|.

10. Bernardeschi D, Pyatigorskaya N, Russo FY, et al. Anatomical, functional and quality-of-life results for mastoid and epitympanic obliteration with bioactive glass s53p4: a prospective clinical study. Clin Otolaryngol 2017;42 (2):387-96 doi: 10.1111/coa.12748[published Online First: Epub Date]|.

11. Silvola JT. Mastoidectomy cavity obliteration with bioactive glass: a pilot study. Otolaryngol Head Neck Surg 2012;147 (1):119-26 doi: 10.1177/0194599812438168[published Online First: Epub Date]|.

12. Merchant SN, Wang P, Jang CH, et al. Efficacy of tympanomastoid surgery for control of infection in active chronic otitis media. Laryngoscope 1997;107 (7):872-7 doi: 10.1097/00005537-19970700000007[published Online First: Epub Date]|.

13. Saleh HA, Mills RP. Classification and staging of cholesteatoma. Clin Otolaryngol Allied Sci 1999;24 (4):355-9 doi: 10.1046/j.1365-2273.1999.00272.x[published Online First: Epub Date]|. 


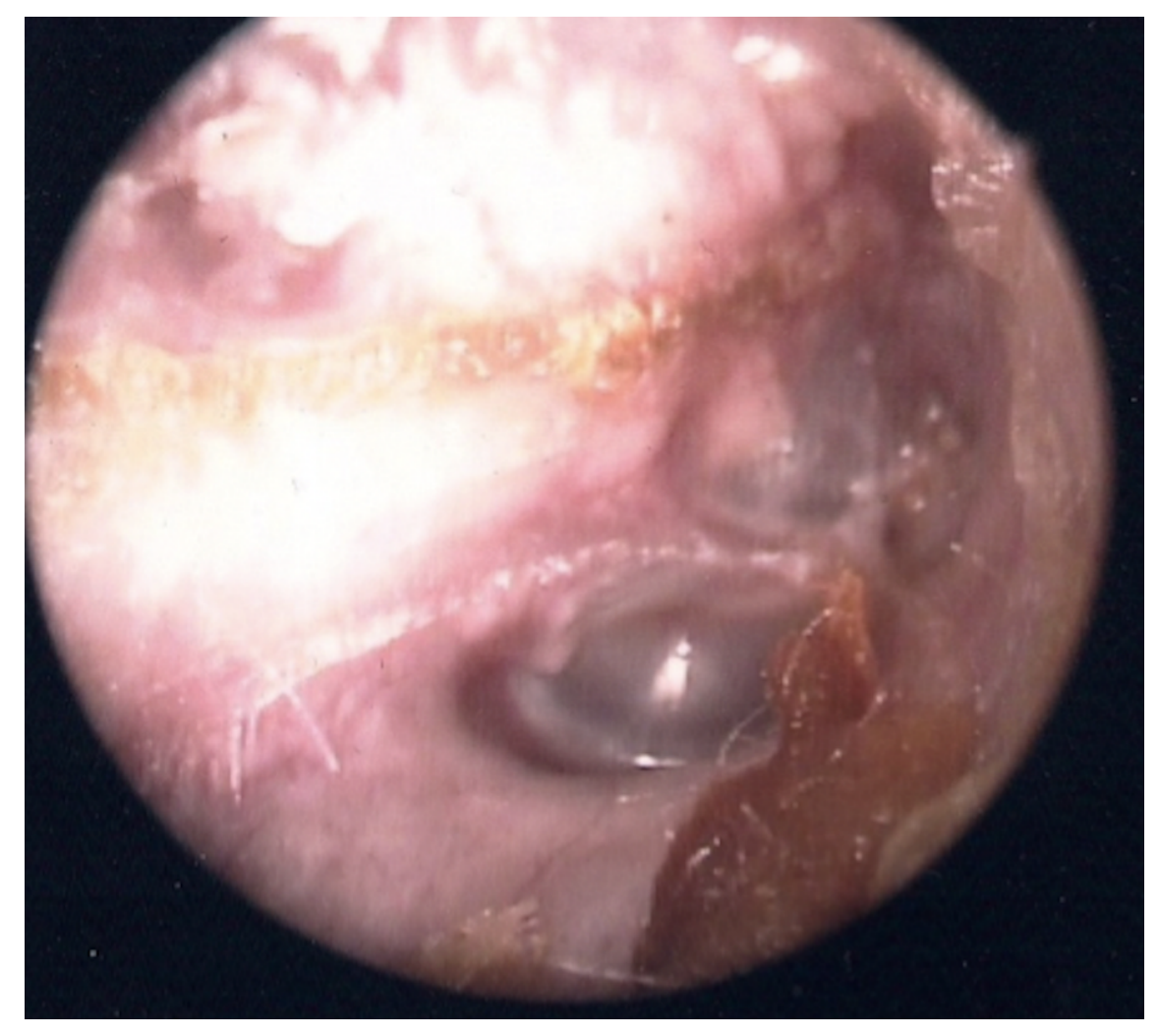




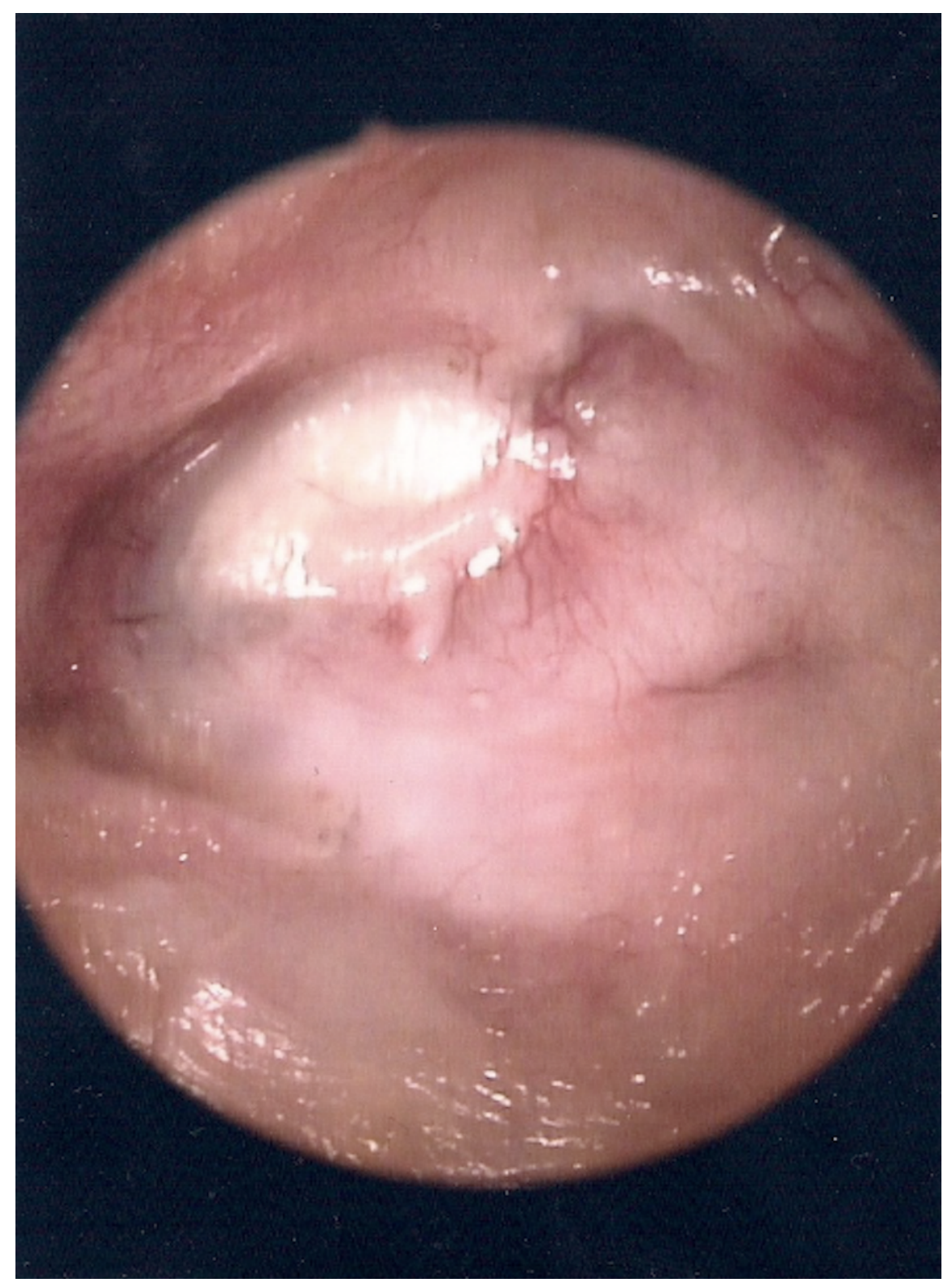

\section{Hosted file}

Figure 1.docx available at https://authorea.com/users/333023/articles/459318-a-multicentreretrospective-case-review-of-outcomes-and-complications-of-s53p4-bioactive-glass-inmastoid-obliteration-surgery

\section{Hosted file}

Figure 2.docx available at https://authorea.com/users/333023/articles/459318-a-multicentreretrospective-case-review-of-outcomes-and-complications-of-s53p4-bioactive-glass-inmastoid-obliteration-surgery

\section{Hosted file}

Table 1.docx available at https://authorea.com/users/333023/articles/459318-a-multicentre- 
retrospective-case-review-of-outcomes-and-complications-of-s53p4-bioactive-glass-inmastoid-obliteration-surgery

\section{Hosted file}

Table 2.docx available at https://authorea.com/users/333023/articles/459318-a-multicentreretrospective-case-review-of-outcomes-and-complications-of-s53p4-bioactive-glass-inmastoid-obliteration-surgery 